www.jmscr.igmpublication.org Impact Factor 5.244

Index Copernicus Value: 83.27

ISSN (e)-2347-176x ISSN (p) 2455-0450

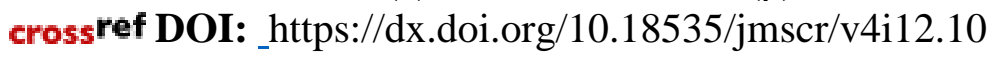

Journal Of Medical Science And Clinical Research

\title{
Free Flap versus Pedicled Flap Reconstruction for Oral Cavity Malignancies: Treatment algorithm based on our experience with 37 cases in our institute
}

\author{
Authors \\ Sagar Gundewar', Al-Iqyan Fidvi², Richa Goyal ${ }^{3}$, Manoj Pawar ${ }^{4}$, \\ Parmeshwar Satpathy ${ }^{5}$ \\ ${ }^{1}$ MBBS, MS (General Surgery), Mch Plastic \& Reconstructive Surgery, Department of Plastic \& \\ Reconstructive Surgery, Grant Government Medical College \& Sir JJ Group of Hospitals \\ ${ }^{2}$ MBBS, MS (General Surgery), Department of Plastic \& Reconstructive Surgery, Grant Government \\ Medical College \& Sir JJ Group of Hospitals \\ ${ }^{3}$ MBBS, MS (General Surgery), Department of Plastic \& Reconstructive Surgery, Grant Government \\ Medical College \& Sir JJ Group of Hospitals \\ ${ }^{4}$ MBBS, MS (General Surgery), Department of Plastic \& Reconstructive Surgery, Grant Government \\ Medical College \& Sir JJ Group of Hospitals \\ ${ }^{5}$ MBBS, MD (PSM), Statitician, Department of Preventive \& Social Medicine, Grant Government Medical \\ College \& Sir JJ Group of Hospitals \\ Corresponding Authors \\ Dr Sagar Gundewar \\ Gokuldas Tejpal Hospital \\ Affiliated to Grant Government Medical College \& Sir JJ Group of Hospitals, Mumbai \\ Email: sagargundewar@gmail.com,+91998750584 \\ Dr Al-Iqyan Fidvi \\ Gokuldas Tejpal Hospital \\ Affiliated to Grant Government Medical College \& Sir JJ Group of Hospitals, Mumbai \\ Email: aliqyan@gmail.com, 9623224466
}

\begin{abstract}
Introduction- The reconstruction of defects after excision of oral malignancy is a challenge. The armamentarium of a reconstructive surgeon includes a plethora of flaps such as microvascular free flaps and regional or local pedicled flaps. The patients in our institution require a holistic approach towards not only reconstruction of the defect but also towards improving their nutritional status within the limits of their socioeconomic background.

Methods: We conducted an epidemiological study of patients with oral malignancy in our institute over a period of 3 years. Thirty seven patients were studied during their course of management from pre-operative preparation to post-operative follow up. The choice of flap used for reconstruction depended on location and volume of defect, seropositive status and ejection fraction of the patients.

Results:- Of the 37 patients studied, there were 28 males and 7 females, with an average age of 49 years. The most common site of involvement was the buccal mucosa (43\%) and most common histopathological type was squamous cell carcinoma (96\%). Microvascular free flaps were used for reconstruction in $72 \%$ of patients and
\end{abstract}


pedicled flaps were used in the remainder. Major complications which occurred with microvascular free flaps were hematoma formation with compromised flap circulation (4 cases), complete flap loss (2 cases), and venous congestion requiring intervention of the flap due to venous thrombosis. No major complications were reported with the pedicled flaps. Other complications included partial flap necrosis, oro-cutaneous fistula formation, wound infection, skin graft loss at the donor site. No significant relation was found between age, body mass index type of flap and the rate of occurrence of complications.

Conclusion:- As compared to pedicled flaps free flaps have superior outcomes in terms of functional rehabilitation and aesthetic appearance. Factors such as old age and low nutritional status do not preclude the use of free tissue transfer in reconstruction. In our opinion factors such as low ejection fraction and seropositive status are better managed with pedicled flaps.

Keywords:- Oral Carcinomas, Free flaps, Pedicled Flaps, Age, Body mass index, Echocardiography.

\section{Introduction}

Malignancies of the oral cavity are those that are located on the tongue, floor of mouth, retro molar trigone, upper and lower gingiva, hard palate, buccal mucosa, and the lips ${ }^{[1]}$. Defects arising after excision are composite in nature and include a variety of structures such as skin, mucosa, soft tissue and bone. The anatomy of the oral cavity is complicated, and each structure plays a specific role in speech, swallowing, and facial expression. In addition, defects in one specific functional unit can affect adjacent structures.

The reconstruction of defects following excision in oral malignancies is a challenging job. The difficulty lies not only in reconstructing the defect both functionally and cosmetically but also a holistic approach of treatment towards the patient. This includes proper understanding of the disease and its peri-operative consequences. All this has to be done within the limits of the patient's socioeconomic bio-profile and social support system.

A typical patient of oral malignancy presenting in our government run tertiary care centre is usually referred from various primary healthcare centres and are usually in the higher stages of malignancy requiring major reconstructive efforts. Most of the patients presenting at our institute are from a low socioeconomic strata and hence are both nutritionally depleted and have a poor financial support. Management of these patients is a herculean task involving their nutritional improvement, oncologic management including both surgical and medical procedures within the constraints of a limited financial background and of course, time. Patients may thus, incur high personal health expenditures and thus may be pushed below poverty line and threaten social stability ${ }^{[2]}$.

The armamentarium of the reconstructive surgeon for defects following excision of oral malignancies includes skin/mucosal grafts, regional or local pedicled flaps and vascularized free tissue transfer ${ }^{[3]}$. Compared to pedicled flaps, microvascular flaps provide greater flexibility in harvesting composite tissues and greater volume of tissues and have thus become the gold standard for extensive reconstructions ${ }^{[4]}$. We present our experience of managing advanced stage oral malignancy cases in our institute. We prefer the use of free flaps over pedicled flaps, however the use of pedicled flaps may be indicated in specific cases. We discuss the factors such as age, BMI and choice of flap and their relation to the rate of occurrence of complications.

\section{Materials and Methods}

Thirty seven patients of oral malignancy were treated over a period of 45 months from February 2013 to November 2016. The patients' socioeconomic history, general demographic data, body mass index and addiction history were documented. A 2D Echocardiogram was done for every patient, and those with an ejection fraction below $45 \%$ were considered for a pedicled flap. Patients were screened for Human Immunodeficiency Virus (HIV), Hepatitis B surface antigen (HbsAg) and Hepatitis $\mathrm{C}(\mathrm{HCV})$. Cases positive for HIV were also considered for pedicled flaps. All patients underwent wide local excision of the 
tumour and neck dissection. The choice of flap for reconstruction of the resultant defect depended on a number of factors including location of the defect, type and amount of tissue resected (skin/mucosa/bone) and preoperative or postoperative radiotherapy. The first choice for reconstruction for all patients was a free flap which included fibular osseo cutaneous flap, anterolateral thigh flap (ALT), radial forearm flap (FRAFF). Pedicled flaps were used for specific indications such as HIV positive patients, low ejection fraction and previously irradiated patients. Depending on the amount of tissue required we used either a deltopectoral plus pectoralis major myocutaneous flap (DP-PMMC) or just a pectoralis major myocutaneous flap. Postoperative monitoring of the free flaps were done as per standard protocols discussed by Salgodo $^{[5]}$ in their seminars. Immediate and late postoperative complications were recorded and managed. Major complications such as haematoma formation and flap congestion were dealt with immediate exploration of the neck and redoing the anastomosis if required. Minor complications such as orocutaneous fistula formation, partial flap necrosis and donor site complications were managed either surgically or conservatively depending on the severity. Tumour characteristics including the stage and histopathological type were documented. Statistical analysis was performed using a commercially available software package, IBM SPSS version 23. Chi Square test was used to test the correlation in rate of occurrence of complications with age, BMI and procedure. Correlation was also calculated for occurrence of complications with a particular procedure.

Patients were followed up after discharge at 1, 3, 6 and 12 months, and 1 yearly thereafter. All patients were given post-operative radiotherapy in the standard doses. Each patient's functional and cosmetic outcome was recorded when the patient came for follow up.

\section{Results}

Of the 37 patients operated at our institute, there were 28 males and 7 females (male to female ratio, 3.6:1) (Table 1). The average age of the patients was 49.8 years (range, 23 - 72 years). There were 31 patients who were above 40 years of age. The average BMI was $19.5 \mathrm{kgm}^{-2}$, ranging from $17 \mathrm{kgm}^{-2}$ to $24 \mathrm{kgm}^{-2}$. All patients had a history of tobacco consumption in some form or another. The mean duration of tobacco use was 10.5 years, ranging from two to 25 years. Some of the cases had history of chewing both betel nut and tobacco. There were four cases of recurrent carcinomas, one of which presented with an exposed implant. The ejection fraction was less than $45 \%$ in five cases. One patient had history of previous radiotherapy following excision of a previous oral carcinoma. There were four cases (10\%) who tested positive for HIV.

Squamous cell carcinoma was the most common histopathologic type (96\%) followed by adenocystic carcinoma (2\%) of the tongue and mucopeidermoid carcinoma (2\%) of the hard palate in the study (Table -2). The sites in oral cavity were deemed according to where the lesion started on history given by the patient. The commonest site of involvement was the buccal mucosa $(43 \%)$ followed by lower gingivobuccal sulcus $(22 \%)$, lower lip (14\%), tongue (11\%), upper gingivobuccal sulcus $(5 \%)$ and the hard palate $(5 \%)$ (Table- 2). Depending on the stage of the disease, wide local excision with neck dissection was planned. Bony resection was done depending on the extent of mandibular or maxillary involvement. A total of 28 cases $(75 \%)$ required bony resections as follows, segmental mandibulectomy in 11 cases $(30 \%)$, hemimandibulectomy in cases $5(14 \%)$, marginal mandibulectomy in 3 cases (8\%), maxillectomy in 2 cases $(5 \%)$ and maxillary plus mandibular resection in 7 cases $(18 \%)$.

The choice of flaps (Table -3) used for reconstruction was microvascular flaps in 26 cases (72\%). These included fibular osseocutaneous flap in $10(28 \%)$ cases, anterolateral thigh flap (ALT) in $10(28 \%)$ cases, radial forearm flap (FRAFF) in 
$6(16 \%)$ cases and a single case of FRAFF and pectoralis major myocutaneous flap (PMMC) (Table-3). The remaining patients were reconstructed using pedicled flaps which included deltopectoral flap with pectoralis major myocutaneous flap (DP-PMMC) or just PMMC in $10(13 \%)$ cases each.

The most common major complication (Table- 4) was hematoma in the neck following reactionary haemorrhage in four cases $(10 \%)$ which led to pedicle compression and ischemic changes in flap. Patients were immediately explored, clots were evacuated and haemostasis was achieved. Next major complication was venous congestion of the flap which was seen in $3(8 \%)$ cases. These were detected within 48 hours and were immediately taken for exploration. There was evidence of venous thrombosis at the anastomotic site in all 3 cases and the anastomosis was redone. Of these cases one was free fibular flap which was successfully salvaged but the other two, a free fibular flap and a free ALT flap could not be salvaged. The flaps were removed and the resultant defects were reconstructed with pedicled flaps (DP+PMMC flaps).

Minor complications were seen in $48 \%$ of cases (Table 5). Orocutaneous fistulas were managed conservatively by withholding oral intake, dressings, keeping the neck drain for a longer period and injection

glycopyrrolates. Complications such as partialflapnecrosis and inset dehiscence were managed operatively with debridement and reinset of the flap.

Donor site complications in the form of graft loss or infection were either managed conservatively with VAC dressings and/or skin grafts depending on the wound status and amount of graft loss.

All patients were referred to the radiation oncologist for radiation therapy after complete wound healing. No significant flap complications were noted during or after radiotherapy period. All patients had acceptable functional and aesthetic outcomes in our series. None of the patients required any additional procedures thereafter.

\begin{tabular}{|c|c|c|c|}
\hline \multicolumn{2}{|c|}{ Characteristic } & Value $(\mathbf{N})$ & $\%$ \\
\hline \multicolumn{2}{|c|}{ Male : Female } & 3.6:1 & \\
\hline \multicolumn{2}{|c|}{ Mean Age } & 49.8 years & \\
\hline \multicolumn{2}{|c|}{ Mean Duration of Tobacco Chewing } & 10.5 years & \\
\hline \multicolumn{2}{|c|}{ Preoperative Radiotherapy } & 1 cases & 3 \\
\hline \multicolumn{2}{|c|}{ Cases of Recurrent Carcinoma } & 4 & \\
\hline \multirow[t]{2}{*}{ BMI } & $<=20 \mathrm{~kg} / \mathrm{m} 2$ & 26 & 71 \\
\hline & $>20 \mathrm{~kg} / \mathrm{m} 2$ & 11 & 29 \\
\hline \multicolumn{2}{|c|}{$\begin{array}{l}\text { Ejection Fraction on 2D } \\
\text { Echocardiography }<45 \%\end{array}$} & 5 & 14 \\
\hline \multicolumn{2}{|c|}{ HIV positive cases } & 4 & 10 \\
\hline
\end{tabular}

\begin{tabular}{|c|c|c|c|c|c|}
\hline \multicolumn{6}{|c|}{ Table 2 -Tumour Profile } \\
\hline \multicolumn{3}{|c|}{ Primary Tumour Site } & \multicolumn{3}{|l|}{ Histopathology } \\
\hline Site & $\mathrm{N}$ & $\%$ & Type & $\mathrm{N}$ & $\%$ \\
\hline $\begin{array}{l}\text { Buccal } \\
\text { Mucosa }\end{array}$ & 16 & 43 & $\mathrm{SCC}$ & 35 & 96 \\
\hline Hard Palate & 2 & 5 & $\begin{array}{l}\text { Adenocystic } \\
\text { Carcinoma }\end{array}$ & 1 & 2 \\
\hline Upper GB & 2 & 5 & \multirow{4}{*}{$\begin{array}{l}\text { Mucoepiderm } \\
\text { oid } \\
\text { Carcinoma of } \\
\text { Hard Palate }\end{array}$} & \multirow[t]{4}{*}{1} & \multirow[t]{4}{*}{2} \\
\hline Lower GB & 8 & 22 & & & \\
\hline Tongue & 4 & 11 & & & \\
\hline Lower Lip & 5 & & & & \\
\hline
\end{tabular}

\begin{tabular}{|c|c|c|}
\hline & $\mathbf{N}$ & $\%$ \\
\hline \multicolumn{3}{|l|}{ Free Flaps } \\
\hline - FRAFF & 6 & 16 \\
\hline - $\quad$ Free ALT & 10 & 28 \\
\hline - FFOCF & 10 & 2 \\
\hline \multicolumn{3}{|l|}{ Pedicled Flaps } \\
\hline - DP-PMMC & 5 & 14 \\
\hline - PMMC & 5 & 14 \\
\hline \multicolumn{3}{|l|}{ Free + Pedicled Flaps } \\
\hline - $\quad$ PMMC + FRAFF & 1 & 2 \\
\hline
\end{tabular}

\begin{tabular}{|c|c|c|c|}
\hline \multicolumn{4}{|c|}{ Table 4 - Major Complications } \\
\hline \multirow{3}{*}{$\begin{array}{c}\text { Major } \\
\text { Complication } \\
\text { (Total) }\end{array}$} & \multirow{3}{*}{$\begin{array}{c}7 \\
(19 \%)\end{array}$} & $\begin{array}{l}\text { Hematoma } \\
\text { formation }\end{array}$ & $\begin{array}{l}4 \\
(11 \%)\end{array}$ \\
\hline & & $\begin{array}{l}\text { Venous } \\
\text { Congestion }\end{array}$ & $\begin{array}{l}1 \\
(3 \%)\end{array}$ \\
\hline & & $\begin{array}{l}\text { Venous } \\
\text { thrombosis with } \\
\text { Total Flap loss }\end{array}$ & $\begin{array}{l}2 \\
(5 \%)\end{array}$ \\
\hline
\end{tabular}




\begin{tabular}{|c|c|c|c|c|c|c|c|}
\hline \multicolumn{8}{|l|}{ Table 5 - Minor Complications } \\
\hline & \multicolumn{2}{|c|}{$\begin{array}{l}\text { Pedicled } \\
\text { Flaps }\end{array}$} & \multicolumn{5}{|c|}{ Free Flaps } \\
\hline & $\mathbf{N}$ & $\%$ & $\begin{array}{r}\text { FRAF } \\
\text { F }\end{array}$ & Free ALT & FFOCF & Total & $\%$ \\
\hline Donor site graft loss & & & 2 & 1 & 2 & 5 & 13 \\
\hline $\begin{array}{l}\text { Donor site wound infection with } \\
\text { wound gape }\end{array}$ & 1 & 3 & - & - & 3 & 3 & 8 \\
\hline Oral fistula & 2 & 5 & 1 & - & 1 & 2 & 5 \\
\hline Partial flap necrosis & 2 & 5 & - & 2 & - & 2 & 5 \\
\hline \multirow[t]{2}{*}{ Inset infection with dehiscence } & 1 & 3 & - & - & - & $\mathbf{0}$ & $\mathbf{0}$ \\
\hline & 6 & 16 & & & & 12 & 32 \\
\hline
\end{tabular}

\begin{tabular}{|c|c|c|c|}
\hline \multicolumn{4}{|c|}{ Table 6 - Relation with age and BMI } \\
\hline Factor & Cases & Complication & $P$ Value \\
\hline $\begin{array}{c}\text { Age }>40 \\
\text { yrs. }\end{array}$ & 31 & 16 & \multirow{2}{*}{0.734} \\
\hline $\begin{array}{c}\text { Age }<40 \\
\text { yrs. }\end{array}$ & 6 & 3 & \\
\hline BMI > 20 & 11 & 6 & \multirow{2}{*}{0.384} \\
\hline BMI $<=20$ & 26 & 14 & \\
\hline \multirow[b]{2}{*}{ Flap type } & Free Flap & 19 & \multirow[b]{2}{*}{0.564} \\
\hline & $\begin{array}{l}\text { Pedicled } \\
\text { Flap }\end{array}$ & 6 & \\
\hline
\end{tabular}

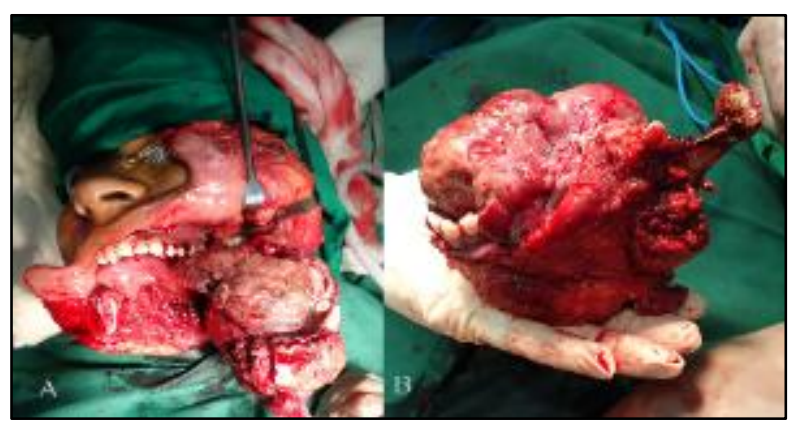

Fig 1:- A case of left buccal mucosal carcinoma post excision showing a extensive composite defect which includes a large external skin deficit, lateral segment of mandible, floor of mouth, part of left oral commissure and buccal mucosa upto the retro malar trigone A) defect size B) excised lesion

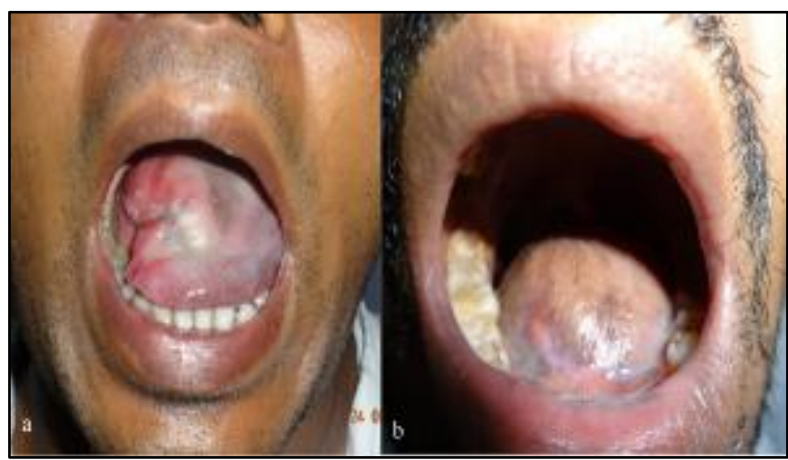

Fig 2:- A case of carcinoma tongue posted for total glossectomy reconstructed using FRAFF

A) preoperative B) postoperative photo after 4 weeks showing a well mucolised flap

\section{Discussion}

Oral cavity cancer ranks among the top ten of the most prevalent malignancies affecting patients worldwide. Squamous cell carcinoma is by far the most common histology for tumours of the oral cavity $^{[1]}, 96 \%$ of cases in our study were squamous cell carcinoma. This concurs with the data from the Tata Memorial Hospital (TMH) tumour registry $2001^{[6]}$. The most common site is the buccal mucosa followed lower gingivobuccal sulcus followed by lower GB sulcus other sites (Table 2). As compared to the data in $\mathrm{TMH}$ tumour registry primary cases of lower lip had higher prevalence in our study, $14 \%$ to $1 \%$ in the TMH registry, while the incidence of primary carcinoma of the tongue was $11 \%$ in our series which was lower as compared to $33 \%$ incidence in the registry. 


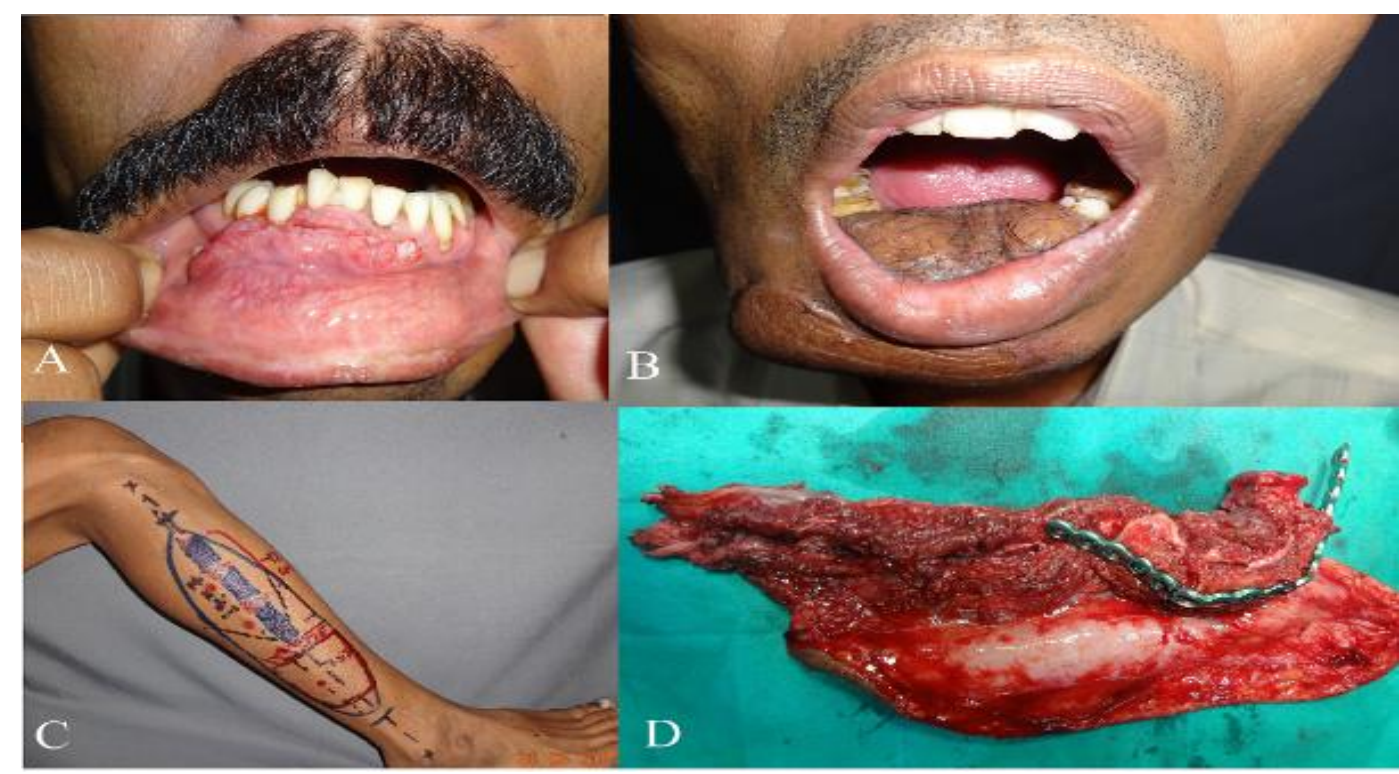

Fig 3:- A case of carcinoma lower lip. WLE done with central segmental mandibulectomy \& reconstructed using free fibular flap. A) Preoperative photo B) postoperative photo 3 weeks C) Preoperative planning of flap showing skin paddle for internal lining, external skin defect and part of flap to be de-epithelized (red). D) Reconstructed fibula before inset

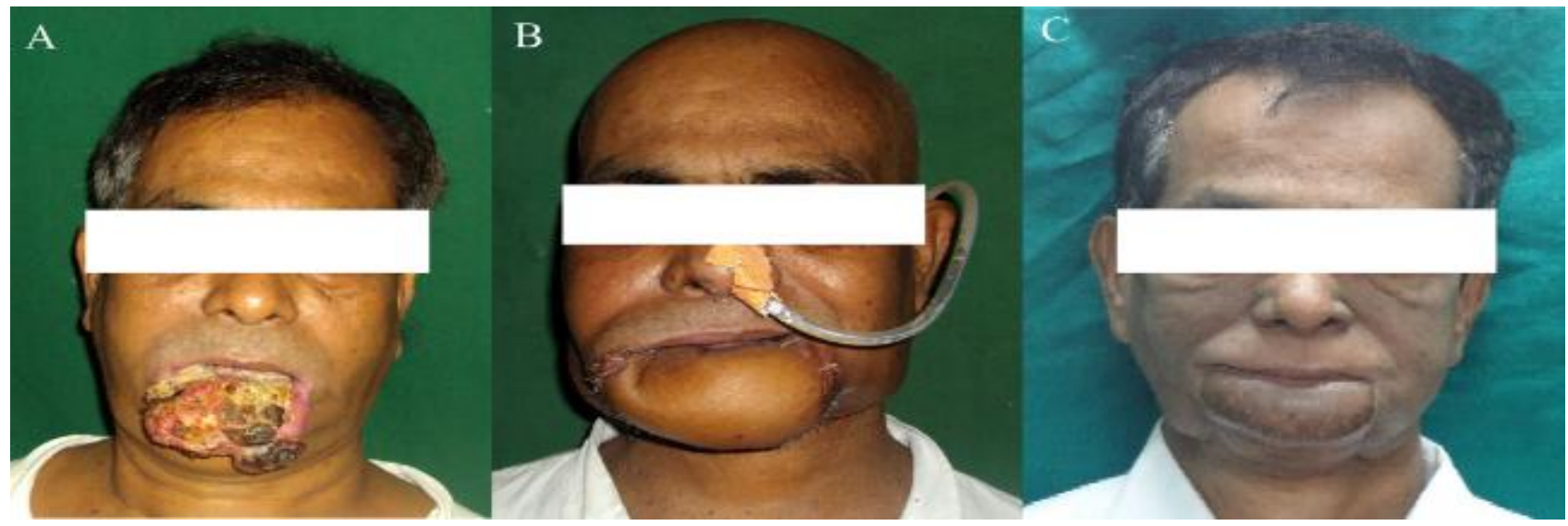

Fig 4:- A case of carcinoma lower lip which was reconstructed using free ALT flap post excision A) Preoperative photo B) Postoperative photo at 2 weeks C) Follow up after radiotherapy showing reduced bulk of flap

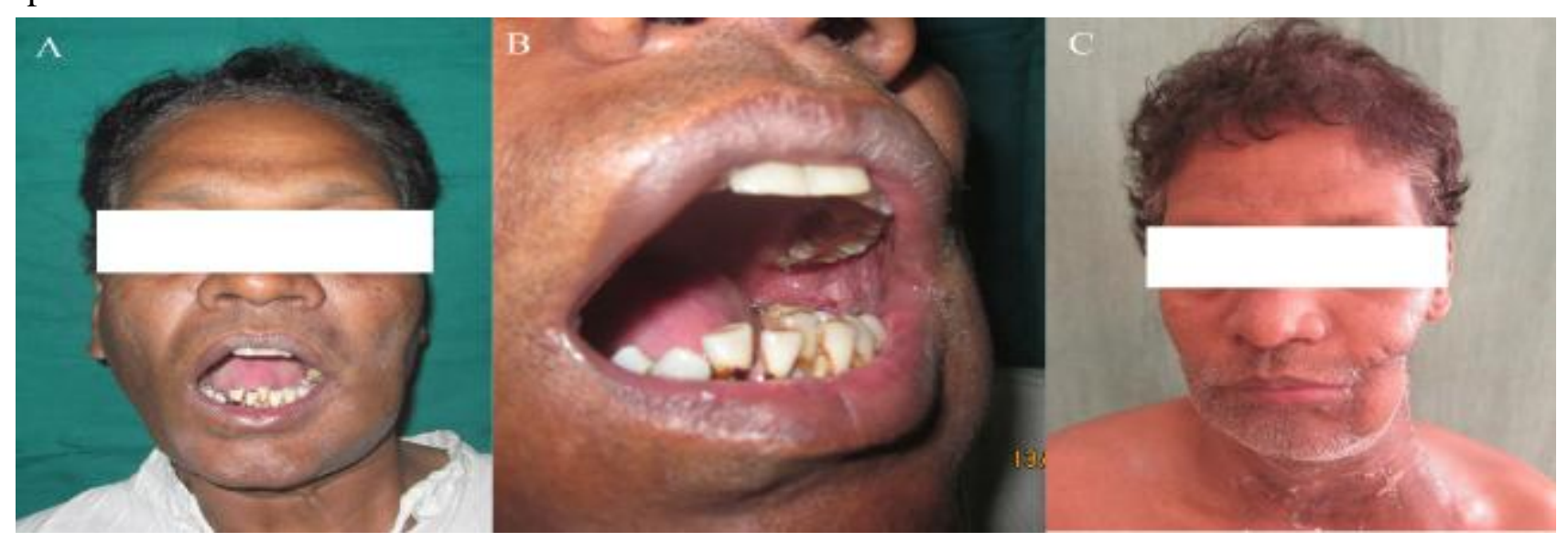

Fig 5:- A case of buccal mucosa on left cheek region. Patient was reconstructed using a pectoralis major myocutaneous flap (PMMC) post excision. A) Preoperative profile photo B) Preoperative lesion in left buccal mucosa C) Postoperative photo after 2 months 
The most common risk factors for oral malignancy are tobacco and alcohol $1^{[7]}$. In southeast Asia including India, the use of betel quid (which involves chewing arious combinations of areca nut, catechu, slaked lime, and tobacco wrapped in a betel leaf poses a significant risk for oral cancer, contributing greatly to the incidence worldwide $^{[8] .}$ As shown by Phukan et al ${ }^{[9]}$, the duration of chewing is directly proportional to the incidence of oral cavity cancer. They also confirmed that the dose response has a more carcinogenic effect as compared to the duration response. The mean duration of tobacco chewing, of patients in our series was 10.5 years. Most of the cases, especially males had started chewing tobacco/ betel quid before the age of 20 years. In India the use of tobacco or its various forms used as 'smokeless tobacco' is to a tune of $59 \%$ in males and $43 \%$ in females. Excessive retention of such substances in the mouth has been proven to increase the risk of oral cavity cancers ${ }^{[10]}$.

Following oncological resection of the oral cavity carcinomas, we are left with extensive bone and soft tissue defects (Figure 1). The goals for reconstruction include reshaping the lower half of the face, maintaining function (mastication, swallowing and speech), a stable wound which does not result in an orocutaneous fistula and quick healing. In addition the flaps used should not cause significant obstruction of the oral cavity or the airway and allow the patient to maintain oral hygiene. Microvascular free flaps have become the first choice for reconstruction of complex defects in the oral cavity ${ }^{[7]}$. While pedicled flaps are used in certain specific indications and as a lifeboat after failure of free flaps.

The specific reconstructive technique is chosen depending on several key considerations. These include location of the defect, amount and type of tissue resected i.e. skin, mucosa and bone. For instance a thin pliable radial artery flap is preferred over a bulky ALT flap for hemiglossectomy in order to preserve tongue mobility (Figure 2). Other factors to keep in mind are any plans for dental restoration including osseointegration, whether patient has received preoperative radiotherapy and whether postoperative radiotherapy is planned.

As the cases in our study were fairly advanced when they presented to us, the post excisional defects were extensive involving some form of mandible resection which included either a marginal, segmental or a hemimandibulectomy. Where a bone defect in the form of segmental or hemimandibulectomy was present, a free fibular osseo cutaneous flap (Figure 3) was preferred over a free ALT flap as has been advised in the metacentric review article by Thomas ${ }^{[1]}$. In our study, out of the $10(27 \%)$ cases in which a segmental mandibulectomy was done 9 cases $(90$ $\%$ ) of these were reconstructed with a free fibular osseo cutaneous flap. In cases of massive defects of maxilla and mandible our preference is to use a bulky flap such as an ALT flap or a combination of two pedicled flaps. The primary aim is to provide bulk which decreases in size after radiotherapy (Figure 4). This has been a consistent finding in many of the studies including that by Higgins ${ }^{[11]}$. A larger skin paddle can be harvested with the free ALT flaps which can be used for both the intraoral as well as the external defects. We preferred using only a soft tissue flap when mandibular/ maxillary resection was limited. Small defects which included only buccal mucosa or just external skin were reconstructed with free radial forearm flap. The flap provides thin, pliable skin, and adequate length of the pedicle which will reach the neck very easily ${ }^{[12]}$.

In patients where the ejection fraction was less than $45 \%$, we preferred to use pedicled flaps over free flaps. Of the ten cases where pedicled flaps were used five patients had an ejection fraction of less than $45 \%$ on $2 \mathrm{D}$ Echo. A low ejection fraction is associated with a low flow in the arterial system. Low ejection fraction is also associated with chronic heart failure which may leads to wide fluctuations in blood pressure ${ }^{[13]}$. We believe that such hypotensive episodes may cause stasis of blood at the anastomotic sites with resultant venous thrombosis leading to failure of flap. Also, if ejection fraction is low any additional stress 
effect placed on circulatory system may have a catastrophic effect on the heart ${ }^{[14]}$.

Another indication for not using free tissue transfer surgery in our institute is when the patient tested sero positive for HIV, HbsAg or HCV. A number of studies have shown that there is a higher risk of exposure when the duration of surgery exceeds three hours, with blood loss more than $300 \mathrm{ml}$ and major vascular or abdominal surgeries ${ }^{[15,16,17]}$.Though operative times may remain comparable while performing a pedicled flap there is lesser degree of dissection and handling of tissues with lesser volume of blood loss and hence lesser risk of exposure for the operating surgeons. There were four cases in our study who were HIV positive and reconstructed with either pectoralis major myocutaneous flap (Figure 5) or deltopectoral plus pectoralis myocutaneous flaps.

Another case where we used a pedicle flap instead of a free flap was in a case of recurrent carcinoma where the patient was irradiated following his previous surgery for excision of buccal mucosal lesion. He had received a very high dose of radiation (more than $90 \mathrm{~Gy}$ ) over a period of 2 weeks. The patient presented to us after 4 weeks of completion of his radiation cycle with recurrent carcinoma of the lower GB sulcus on the same side as the previous lesion. Although free tissue transfer can be used in a previously irradiatedarea, these patients have a higher risk of complications such as flap loss, infections and poor healing of tissues. Following radiotherapy there are pathologic changes in the form of endarteritis, sub endothelial fibrosis, chronic ischemia and local coagulation disorders at the recipient site which leads to increased chances of thrombosis in the blood vessels especially following microvascular anastomosis. There is also difficulty in dissection and identification of vessels for anastomosis ${ }^{[18]}$. Several studies have shown that there is a dose dependent relation of flap loss and other complications with preoperative radiotherapy ${ }^{[19]}$. As the patient had received a very high dose of radiation previously, we felt that a pedicled flap would give a more predictable outcome than a free flap.

Major complications were seen in 7 (19\%) cases. These occurred only in those cases which were reconstructed using free flaps. No major complication in the form flap loss was seen in pedicled flaps. Complete flap loss was seen in two (5\%) cases following venous thrombosis event after attempts to salvage the flaps A single cases which had developed venous congestion was successfully salvaged. Flap loss following venous thrombosis is the most common reported complication following a free tissue transfer surgery ${ }^{[20]}$. The pattern of occurrence of complications in our series is comparable with that of a study by Tarsitano ${ }^{\text {[21] }}$ who also reported donor site morbidity as a major overall complication followed by flap necrosis, inset dehiscence.

There was no significant correlation between the occurrence of complications and the age of the patient (table - 6) which also been reported by various other authors ${ }^{[22,23]}$.Thus microvascular reconstruction can be considered as a safe procedure in elderly patients with oral cavity cancer without significant risk of flap failure or surgical complications.

There was no correlation between the incidence of complications and the BMI of the patients (table 6). In contrast a study by Yang ${ }^{[25]}$, showed a higher incidence of fistula formation and flap infection in cases with lower BMI $<20 \mathrm{~kg} / \mathrm{m} 2$ as compared to $>20 \mathrm{~kg} / \mathrm{m} 2$. Perioperative nutritional support is an important factor in decreasing recipient and donor site morbidity. Early institution of oral or nasogastric tube feeding with an increased protein (particularly egg) and multivitamins helps in preventing infections and other donor site morbidities. Post-operative blood transfusion was rarely given except when the haemoglobin was less than 9 gm $\%$. Whole blood transfusion was preferred over packed cell volume because of the haematocrit raising tendency of the latter which can predispose to cell aggregation and thrombosis at the anastomotic site. 
Functional results are difficult to assess in a heterogeneous oral cancer population with defects including different types of tissues. The four $(11 \%)$ cases of carcinoma tongue which were reconstructed using FRAFF had hyper nasality of voice on follow up. There were no swallowing difficulties or complaints of aspiration of food in these patients. Nine cases which were reconstructed with a free fibular flap on follow up after radiotherapy showed no oral incompetence or speech difficulties. This was not the case when mandible was removed in the form of either anterior segmental or a hemimandibulectomy and defect was reconstructed using soft tissue flaps only. The five cases of carcinoma lip complained of oral incompetence immediately after surgery which gradually lessened after radiotherapy. The quality of speech was occasionally misunderstood or could be understood only when the context of conversation was known to the listener. Cases of carcinoma involving buccal mucosa which after resection had intact oral commissures and a lip seal was preserved mainly had some difficulties in deglutition which resolved after radiotherapy and on late follow up. None of the cases in our series required ant secondary procedures to improve function or facial aesthetics.

\section{Conclusion}

Patients presenting to our institute have advanced stage malignancies, poor nutritional status and low socioeconomic backgrounds. Extensive composite defects of the oral cavity are created after tumour resection in these patients which require complex reconstructive surgical procedures as well as boosting their nutritional status and without placing additional financial burden on their family. Currently free flap reconstruction for oral cavity defects following excision in oral carcinomas is the first option as it gives the most reliable result. It provides versatility of tissues for composite defects and give superior aesthetic and functional outcomes in terms of oral competence and speech. Pedicled flaps are reserved for specific indications such as patient with low ejection fraction, seropositive status and previous history of radiation.

Based on the experience in our institute we have developed algorithms for flap selections so as to consistently reliable results with low complications and minimal donor site morbidity.

\section{References}

1. Thomas JO, Jeffrey NM. Current Management of Advanced Resectable Oral Cavity Squamous Cell Carcinoma. Clinical and Experimental Otorhinolaryngology 2011;4:1-10.

2. SreeVidya Krishna Rao, Gloria Mejia, Kaye Roberts-Thomson, Richard Logan. Epidemiology of Oral Cancer in Asia in the Past Decade- An Update (2000-2012). Asian Pacific journal of cancer prevention 2013;14(10):5567-77.

3. Hurvitz KA, Kobayashi M, Evans GR. Current options in head and neck reconstruction. Plast Reconstr Surg 2006;118: 122e-33e.

4. Yadav P. Reconstructive surgery in oral cancers. Indian J PlastSurg 2007;40:22-27.

5. Christopher J. Salgado, Harvey Chim, Shayla Schoenoff, Samir Mardini. Postoperative Care and Monitoring of theReconstructed Head and Neck Patient. Seminars in plastic surgery 2010;24.

6. TMH Cancer Registry. Published by the Tata Memorial Hospital: Mumbai 2001.

7. Chiu-mingHo, Vana SH Chan, Mingshiaw Cheng, Wing-yung Cheung. Microvascular Free Flaps: Experience in KwongWah Hospital. Medical Bulletin 2007; 12 .

8. Nair U, Bartsch H, Nair J. Alert for an epidemic of oral cancer due to use of the betel quid substitutes gutkha and pan masala: a review of agents and causative mechanisms. Mutagenesis. 2004 Jul;19(4): 251-62.

9. Phukan RK, Ali MS, Chetia CK, Mahanta J. Betel nut and tobacco chewing; potential risk factors of cancer of oesophagus in 
Assam, India. 2001;85(5):661-667. doi:10.1054/bjoc.2001.1920. British Journal of Cancer

10. Gupta PC, Ray CS Smokeless tobacco and health in India and South Asia. Respirology 2003; 8: 419-431.

11. Higgins KM, Erovic BM, Ravi A, Yeung $\mathrm{R}$, Lee JW, Yao C et al. Volumetric changes of the anterolateral thigh free flap following adjuvant radiotherapy in total parotidectomy reconstruction. The Laryngoscope 2012;122: 767-772. doi: 10.1002/lary.22509

12. Shibahara T, Mohammed AF, Katakura A, Nomura T. Long-term results of free radial forearm $B$ ap used for oral reconstruction: Functional and histological evaluation. J Oral MaxillofacSurg 2006;64:1255-60.

13. Gottesman RF, Grega MA, Bailey MM, et al. Association between hypotension, low ejection fraction and cognitive performance in cardiac patients. Behavioural neurology 2010;22(1-2):6371. doi:10.3233/BEN-2009-0261.

14. James L. Robotham, Masao Takata, Michael Berman, Yasuhiko Harasawa. Ejection Fraction Revisited. Anesthesiology 1991;74:172-183.

15. Gerberding JL, Littell C, Tarkington A, Brown A, Schecter WP. Risk of exposure of surgical personnel to patients' blood during surgery at San Francisco General Hospital. N Engl J Med. 1990 Jun;322(25):1788-93.

16. Popejoy SL,FryDE.Blood contact and exposure in the operating room. Surg Gynecol Obstet. 1991 Jun; 172(6):480-3.

17. Quebbeman EJ, Telford GL, Hubbard S, Wadsworth K, Hardman B, Goodman H, Gottlieb MS. Risk of blood contamination and injury to operating room personnel. Ann Surg. 1991 Nov;214(5):614-20.

18. ChaudhariCharudatta. Reconstruction in previously irradiated patients. . Indian J PlastSurg 2007;40:60-64
19. Herle, P., Shukla, L., Morrison, W. A. and Shayan, R. Preoperative radiation and free flap outcomes for head and neck reconstruction: a systematic review and meta-analysis. ANZ J Surg 2015;85: 121127. doi:10.1111/ans.12888

20. Steven PD, Mark WC, Andrew LK. Anatomy of Free Flap Failures: Dissection of a Series. Modern Plastic Surgery 2013;3:89-95.

21. Tarsitano A, Pizzigallo A, Sgarzani R, Oranges $\mathrm{Cm}$, Cipriani R, Marchetti C. Head and neck cancer in elderly patients: is microsurgical free-tissue transfer a safe procedure?. ActaOtorhinolaryngologicaItal ica 2012;32(6):371-375.

22. Pompei S, Tedesco M, Pozzi M. Age as a risk factor in cervicofacial reconstruction. J ExpClin Cancer Res 1999;18:209-12.

23. Bridger AG, O'Brien CJ, Lee K. Advanced patient age should not preclude the use of free flap reconstruction for head and neck cancer. Am J Surg 1994; 168:425-8.

24. Yang Hoi Heo, Shunjiro Yagi, Kazuhiro Toriyama, Keisuke Takanari, Yasushi Fujimoto, Naoki Nishioet al. Relationship between BMI and Postoperative Complications with Free Flap in Anterolateral Craniofacial Reconstruction. Plast ReconstrSurg Glob Open 2016;4:e636; doi: 10.1097. 